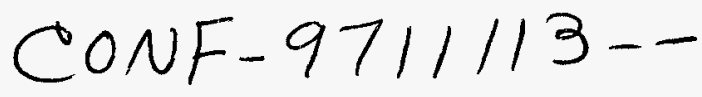

\title{
PROMPT MUON-INDUCED FISSION: A PROBE FOR NUCLEAR FRICTION IN LARGE-AMPLITUDE COLLECTIVE MOTION
}

\author{
VOLKER E. OBERACKER, A. SAIT UMAR \\ Department of Physics \& Astronomy, Vanderbilt University, \\ Nashville, TN 37235, USA \\ E-mail:vvolker.e.oberacker@vanderbilt.edu \\ JACK C. WELLS, MICHAEL R. STRAYER \\ Center for Computational Sciences \& Physics Division, \\ Oak Ridge National Laboratory, \\ Oak Ridge, TN 37831-6373, USA \\ JOACHIM A. MARUHN \\ Institut für Theoretische Physik, Universität Frankfurt, \\ D-60054 Frankfurt am Main, Germany \\ PAUL-GERHARD REINHARD \\ Institut für Theoretische Physik, Universität Erlangen, \\ D-91058 Erlangen, Germany
}

\begin{abstract}
Excited muonic atoms in the actinide region may induce prompt fission by inverse internal conversion, i.e. the excitation energy of the muonic atom is transferred to the nucleus. We solve the time-dependent Dirac equation for the muonic spinor wave function in the Coulomb field of the fissioning nucleus on a 3-dimensional lattice and demonstrate that the muon attachment probability to the light fission fragment is a measure of the nuclear energy dissipation between the outer fission barrier and the scission point.
\end{abstract}

\section{Introduction}

The goal of this project is to use a leptonic probe $\left(\mu^{-}\right)$to study the energy dissipation ("friction") in large-amplitude collective nuclear motion. In this context, nuclear friction is defined as the irreversible flow of energy from collective to intrinsic single-particle motion. There are essentially two different mechanisms that may lead to energy dissipation: "one-body friction", i.e. collisions of the nucleons with the moving walls of the self-consistent mean field and two-body collisions between the nucleons ("two-body friction"). A fundamental and still unresolved question in nuclear many-body theory is the role played by these two energy dissipation mechanisms and the amount of energy dissipation in both hot and cold fission. At this conference, Dr. Back ${ }^{1}$

\section{RECEIVED MAR 061999 \\ O STI}
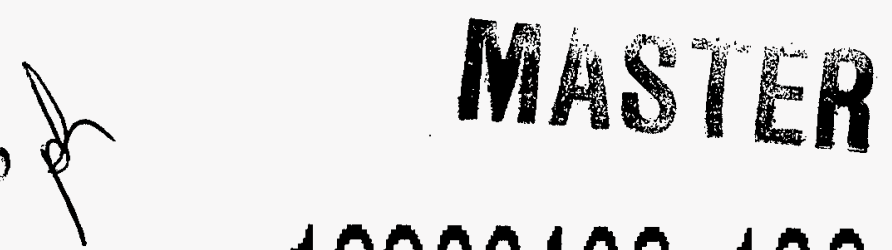
presented data that show the need to invoke a finite amount of viscosity to understand the fission of hot nuclei, and Prof. Itkis ${ }^{2}$ pointed out that one-body dissipation is the most likely mechanism for explaining the fission data.

Following the irradiation of a target with a $\mu^{-}$beam the muons are captured into high-lying atomic states and form an excited muonic atom. Inner shell transitions may proceed without emission of radiation via inverse internal conversion, i.e. the muonic excitation energy is transferred to the nucleus. In actinides, the $2 p \rightarrow 1 s$ and the $3 d \rightarrow 1 s$ muonic transitions result in excitation of the nuclear giant dipole and giant quadrupole resonance, respectively, which act as doorway states for fission. The nuclear excitation energy is typically between 6.5 and $10 \mathrm{MeV}$. Since the muon is not annihilated it can be used to study the fission dynamics.

The prompt muon-induced fission process is most easily understood in terms of a "correlation diagram", i.e. one plots the binding energies of the transient muonic molecule as a function of the internuclear distance ${ }^{3}$. At present, little is known about the nuclear viscosity between the outer fission barrier and the scission point. If the nuclear fission process is slow (large friction), the muon will stay in the lowest molecular energy level $1 s \sigma$ throughout the fission process and emerge in the $1 s$ bound state of the heavy fission fragment. On the other hand, if the nuclear motion is relatively fast (low friction) there is a nonvanishing probability that the muon may be transferred to higher-lying molecular orbitals, e.g. the $2 p \sigma$ level, from where it will end up attached to the light fission fragment. Hence, theoretical studies of the muon-attachment probability to the light fission fragment, $P_{L}$, in combination with experimental data can be utilized to determine the amount of friction.

\section{Outline of the Theory}

For the dynamical description of the muonic wavefunction during prompt fission, the electromagnetic coupling between muon and nucleus $\left(-e \gamma_{\mu} A^{\mu}\right)$ is dominant; the weak interaction is negligible. Because of the nonrelativistic motion of the fission fragments the electromagnetic interaction is dominated by the Coulomb interaction

$$
A^{0}(\mathbf{r}, t)=\int d^{3} r^{\prime} \frac{\rho_{\mathrm{nuc}}\left(\mathbf{r}^{\prime}, t\right)}{\left|\mathbf{r}-\mathbf{r}^{\prime}\right|}
$$

The muonic binding energy in the ground state of an actinide muonic atom amounts to 12 percent of the muonic rest mass; hence nonrelativistic calculations, while qualitatively correct, are limited in accuracy. Several theory groups have demonstrated the feasibility of such calculations ${ }^{4,5}$ which are based on 


\section{DISCLAIMER}

This report was prepared as an account of work sponsored by an agency of the United States Government. Neither the United States Government nor any agency thereof, nor any of their employees, makes any warranty, express or implied, or assumes any legal liability or responsibility for the accuracy, completeness, or usefulness of any information, apparatus, product, or process disclosed, or represents that its use would not infringe privately owned rights. Reference herein to any specific commercial product, process, or service by trade name, trademark, manufacturer, or otherwise does not necessarily constitute or imply its endorsement, recommendation, or favoring by the United States Government or any agency thereof. The views and opinions of authors expressed herein do not necessarily state or reflect those of the United States Government or any agency thereof. 
the time-dependent Schrödinger equation

$$
\left[-\frac{\hbar^{2}}{2 m} \nabla^{2}-e A^{0}(\mathbf{r}, t)\right] \psi(\mathbf{r}, t)=i \hbar \frac{\partial}{\partial t} \psi(\mathbf{r}, t) .
$$

Recently, we have developed a numerical algorithm to solve the relativistic problem on a three-dimensional Cartesian mesh ${ }^{3,6,7}$. The time-dependent Dirac equation for the muonic spinor wave function in the Coulomb field of the fissioning nucleus has the form

$$
H_{\mathrm{D}}(t) \psi(\mathbf{r}, t)=i \hbar \frac{\partial}{\partial t} \psi(\mathbf{r}, t),
$$

where the Dirac Hamiltonian is given by

$$
H_{\mathrm{D}}(t)=-i \hbar c \alpha \cdot \nabla+\beta m c^{2}-e A^{0}(\mathbf{r}, t) .
$$

Our main task is the solution of the Dirac equation for the muon in the presence of a time-dependent external Coulomb field $A^{0}(\mathbf{r}, t)$ which is generated by the fission fragments in motion.

Because the nuclear excitation energy exceeds the fission barrier by several $\mathrm{MeV}$, it is justified to treat the fission dynamics classically (no barrier tunneling). We describe the fission path by a collective coordinate $R$. The classical collective nuclear energy has the form

$$
E_{\mathrm{nuc}}=\frac{1}{2} B(R) \dot{R}^{2}+V_{\mathrm{fis}}(R)+E_{\mu}(R) .
$$

An empirical double-humped fission potential $V_{\text {fis }}(R)$ is utilized ${ }^{8}$ which is smoothly joined with the Coulomb potential of the fission fragments at large $R$. Note that the muon and fission dynamics are coupled via the instantaneous muonic binding energy $E_{\mu}(R)$

$$
E_{\mu}(R(t))=\left\langle\psi(\mathbf{r}, t)\left|H_{\mathrm{D}}(t)\right| \psi(\mathbf{r}, t)\right\rangle
$$

which depends on the fission coordinate; this results in an augmentation of the fission barrier. In addition, we introduce a linear friction force which acts between the outer fission barrier and the scission point to account for energy dissipation. In this case, the dissipation function $D$ is a simple quadratic form in the velocity

$$
\dot{E}_{\text {nuc }}(t)=-2 D=-f \dot{R}^{2}(t) .
$$

The adjustable friction parameter $f$ determines the dissipated energy; it is the only unknown quantity in the theory. 


\section{Lattice Representation and Numerical Algorithm}

We solve the time-dependent Dirac equation (3) on a 3-dimensional Cartesian lattice using the Basis-Spline collocation method. The Basis-Spline functions $B_{i}^{M}(x)$ are piecewise-continuous polynomials of order $(M-1)$. They represent generalizations of finite elements which are B-splines with $M=2$. In the present calculations we employ B-Splines of order $M \geq 5$. Since the spinor wave function is represented by polynomials of high order, very high accuracy can be achieved with a modest number of lattice points. The Dirac spinor components $\psi^{(p)}$ are represented on a rectangular Cartesian lattice $\left(x_{\alpha}, y_{\beta}, z_{\gamma}\right)$ with $\left(N_{x}, N_{y}, N_{z}\right)$ grid points. The four Dirac spinor components $\psi^{(p)}, p=$ $(1, \cdots, 4)$ are expanded in terms of a product of Basis-Spline functions

$$
\psi^{(p)}\left(x_{\alpha}, y_{\beta}, z_{\gamma}, t\right)=\sum_{i, j, k} B_{i}\left(x_{\alpha}\right) B_{j}\left(y_{\beta}\right) B_{k}\left(z_{\gamma}\right) \psi_{(p)}^{i j k}(t)
$$

i.e. the lattice representation of the spinor wave function is a vector with $N=4 \times N_{x} \times N_{y} \times N_{z}$ complex components. For the muon-induced fission problem we use a rectangular lattice of dimension $67 \mathrm{fm} \times 67 \mathrm{fm} \times 146 \mathrm{fm}$ with a uniform lattice spacing $\Delta x=1.5 \lambda_{c}=2.8 \mathrm{fm}$, where $\lambda_{c}=\hbar /\left(m_{\mu} c\right)$ denotes the reduced muon Compton wavelength. The total number of lattice points is given by $N_{x}=25, N_{y}=25, N_{z}=53$ resulting in a vector dimension $N=1.3 \times 10^{5}$. It is impossible to store $H_{\mathrm{D}}$ in memory, since this would require the storage of $N^{2}$ complex double-precision numbers. Even for our moderate lattice size one obtains $N^{2}=1.7 \times 10^{10}$. Hence, we must resort to iterative methods for the solution of the matrix equation which do not require the storage of $H_{\mathrm{D}}$.

We solve the time-dependent Dirac equation in two steps: first, we consider the static Coulomb problem at time $t=0$, i.e. the muon bound to an actinide nucleus

$$
H_{\mathrm{D}}(t=0) \psi_{\mathrm{gs}}=E_{\mathrm{gs}} \psi_{\mathrm{gs}} .
$$

This static problem is solved by an iterative procedure (damped relaxation method $\left.{ }^{3,6}\right)$. The second part of our numerical procedure is the solution of the time-dependent Dirac equation (3) by a Taylor-expansion of the propagator. For an infinitesimal time step $\Delta t$ we find

$$
\psi(t+\Delta t)=U(t+\Delta t, t) \psi(t) \approx\left(1+\sum_{n=1}^{N} \frac{(-i H \Delta t)^{n}}{n !}\right) \psi(t) .
$$

We have thus reduced the original problem to a series of (matrix) $\times$ (vector) operations which can be executed with high efficiency on vector or parallel supercomputers without explicitly storing the matrix in memory. Typical runs 
on a CRAY-YMP-C90 supercomputer utilize between 1,200-1, 900 time steps with a step size $\Delta t=1.5 \tau_{c}=9.3 \times 10^{-24} \mathrm{~s}$, where $\tau_{c}=\lambda_{c} / c$ denotes the reduced muon Compton time.

\section{Numerical Results}

In the following we present results for prompt fission of ${ }_{93}^{237} \mathrm{~Np}$ induced by the $3 d \rightarrow 1 s(9.5 \mathrm{MeV})$ radiationless muonic transition. Fig. 1 shows the muon position probability density for a fission fragment separation $R=43 \mathrm{fm}$; the calculation was carried out for a fission fragment mass asymmetry $\xi=A_{H} / A_{L}=1.10$ with zero friction $(f=0)$. As expected, the muon sticks predominantly to the heavy fragment, but for this small mass asymmetry the muon attachment probability to the light fission fragment, $P_{L}$, is quite sizable.

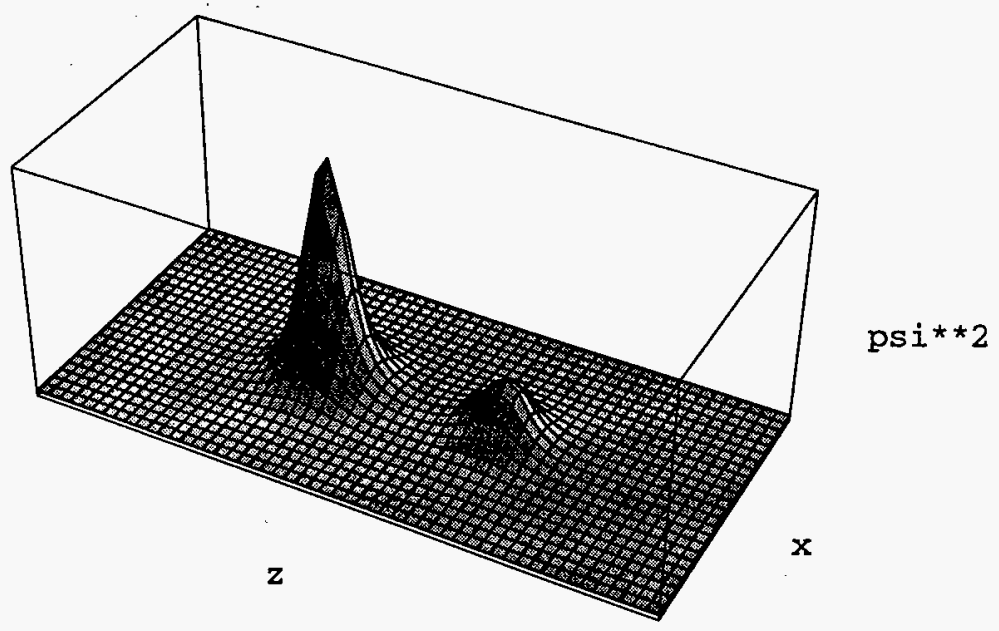

Figure 1: Muon position probability density during fission.

As in all lattice calculations, we need to demonstrate convergence of our results in terms of the lattice size and lattice spacing. Fig. 2 shows the asymptotic muon attachment probability $P_{L}$ as a function of the total number of lattice points; it is apparent that the probabilities have indeed converged.

Fig. 3 shows that $P_{L}$ depends strongly on the fission fragment mass asymmetry. This is easily understood: for equal fragments we must have $P_{L}=0.5$, and for large mass asymmetry it is energetically favorable for the muon to be bound to the heavy fragment, hence $P_{L}$ will be small.

In Fig. 4 we examine the dependence of $P_{L}$ on the dissipated nuclear 


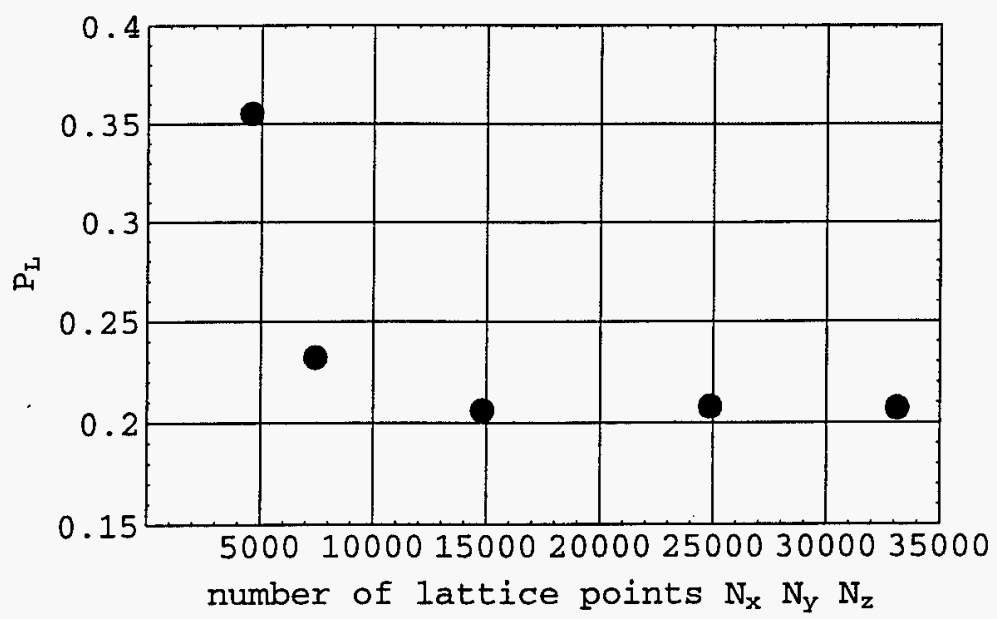

Figure 2: Demonstration of numerical convergence of muon attachment probability for ${ }_{93}^{237} \mathrm{~Np}$. Calculations use a mass asymmetry of 1.10 and zero friction.

energy, $E_{\text {diss }}$, during fission. In our model, friction takes place between the outer fission barrier and the scission point. When the dissipated energy is computed from equation (7) we find an almost linear dependence of the muon attachment probability on $E_{\text {diss }}$; unfortunately, the dependence is not as strong as one might have hoped for.

\section{Comparison with Experimental Data}

Mean lifetimes of muons bound to fission fragments of several actinides have been measured by Schröder et al. ${ }^{9}$. From the K-shell of the muonic atom, the muon decays through various weak interaction processes at a characteristic rate $\lambda=\lambda_{0}+\lambda_{c}$, where $\lambda_{0}=\left(2.2 \times 10^{-6} s\right)^{-1}$ denotes the free leptonic decay rate for the decay process $\mu^{-} \rightarrow e^{-}+\overline{\nu_{e}}+\nu_{\mu}$ and $\lambda_{c}$ the nuclear capture rate; $\lambda_{c}$ depends upon the charge and mass of the fission fragment. From the observed lifetime $\tau_{\mu}=1.30 \times 10^{-7} s$ it was deduced that the muon is predominantly captured by the heavy fragment with a probability $P_{H} \geq 0.9$. It must be emphasized that this number represents an integral over the whole fission mass distribution and, hence, cannot easily be compared to our theory.

More recent experiments have been carried out by Risse et al ${ }^{11}$ using the muon beam of the $\pi E 3$ channel at the Paul Scherrer Institute (PSI) in Switzerland. For this purpose, a fission chamber has been inserted into the electron spectrometer SINDRUM I. The incident muons are detected by a 


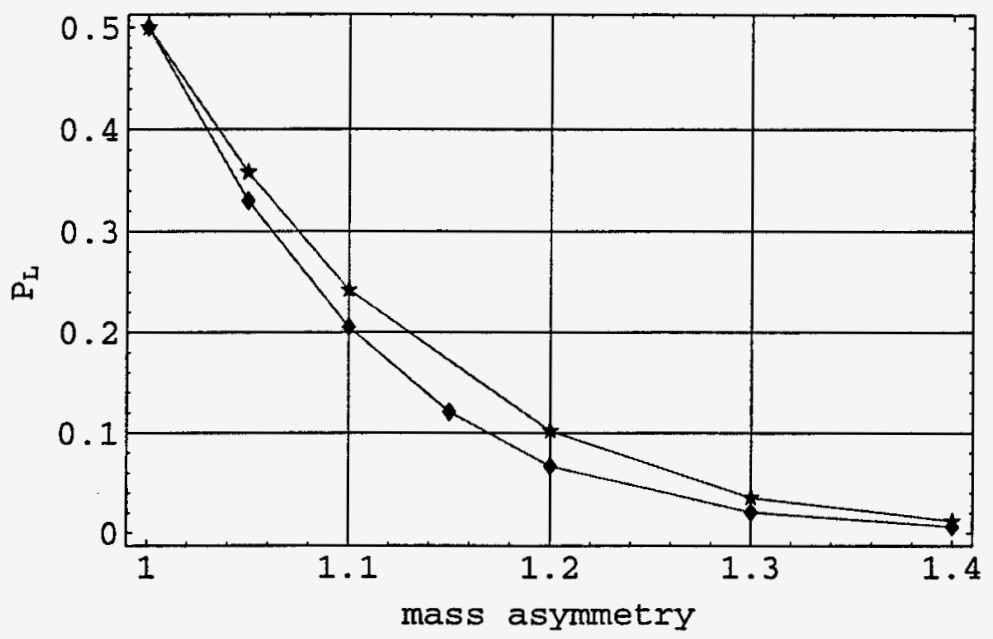

Figure 3: Muon attachment to light fission fragment vs. fission fragment mass asymmetry. upper curve: ${ }_{92}^{238} \mathrm{U}$, lower curve: ${ }_{93}^{237} \mathrm{~Np}$. The calculations assume zero friction.

scintillation counter. An event is defined by a $\left(\mu^{-}, f_{1} f_{2} e^{-}\right)$coincidence where the fission fragments are observed in prompt and the muon decay electrons in delayed coincidence with respect to the incident muon. The magnetic field of the electron spectrometer allows for a three-dimensional reconstruction of the electron trajectories; thus, it is possible to attribute the muon decay electrons to a particular fission fragment.

In this way, muon attachment probabilities $P_{L}$ have been measured for several light fission fragment mass bins $A_{L}$. The experimental data are given in Table 1. Because the mass bins are relatively broad and because $P_{L}$ depends strongly on the mass asymmetry, we cannot assume that $P_{L}$ remains constant within the bin. Hence, in order to be able to compare our theory with the experimental data, we have to multiply our theoretical values depicted in Fig. 4 with a weighting factor that accounts for the measured relative mass distribution $^{11}$ of the prompt fission events within this mass bin. We then integrate these results over the appropriate mass range.

The resulting theoretical values for $P_{L}$ are given in the last column of Table 1. It is apparent that our theory agrees rather well with experiment. Because of the size of the error bars in the experiment and because of the weak dependence of the theoretical values of $P_{L}$ on the dissipated energy, it is not possible to extract very precise information about the amount of energy dissipation. However, by comparing our theoretical result for the mass bin 


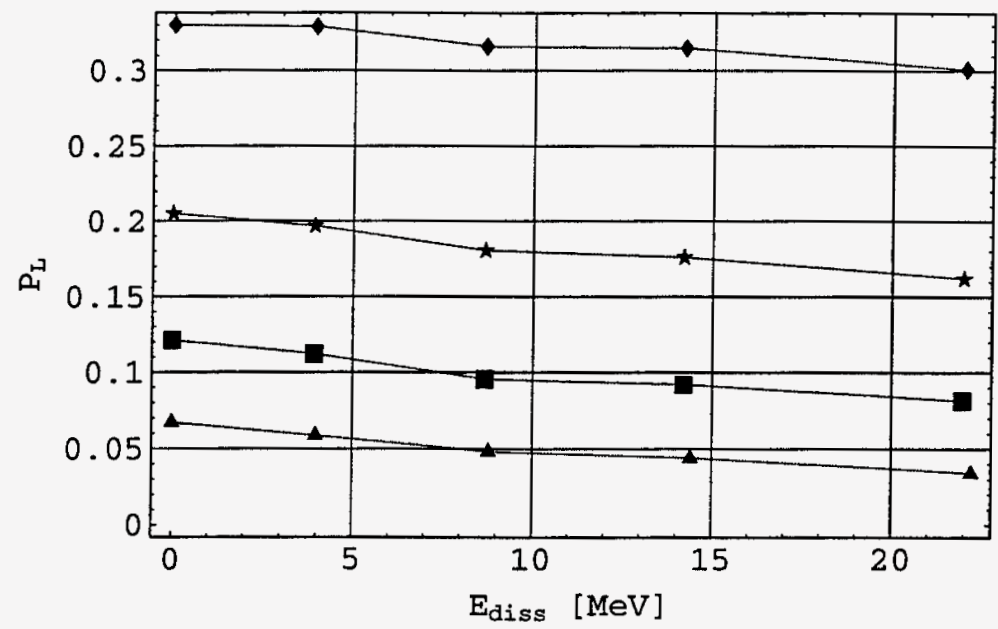

Figure 4: Probability of muon attachment to the light fission fragment as a function of nuclear energy dissipation for ${ }_{93}^{237} \mathrm{~Np}$. The various curves are for fragment mass asymmetries $\xi=1.05,1.10,1.15,1.20$, respectively.

Table 1: Muon-attachment probabilities to the light fission fragment, $P_{L}$, for ${ }^{237} \mathrm{~Np}\left(\mu^{-}, f\right)$.

\begin{tabular}{|c|c|c|c|}
\hline mass bin $A_{L}$ & mass asymmetry & $P_{L}(\exp )$ & $P_{L}($ theo $)$ \\
\hline $118.5 \rightarrow 111.5$ & $1.000 \rightarrow 1.126$ & $(25.5 \pm 8.5) \times 10^{-2}$ & $\begin{array}{c}25.9 \times 10^{-2}, E_{\text {diss }}=0 \mathrm{MeV} \\
22.3 \times 10^{-2}, E_{\text {diss }}=22 \mathrm{MeV}\end{array}$ \\
\hline $111.5 \rightarrow 104.5$ & $1.126 \rightarrow 1.268$ & $(9.7 \pm 2.6) \times 10^{-2}$ & $6.62 \times 10^{-2}, E_{\text {diss }}=0 \mathrm{MeV}$ \\
\hline
\end{tabular}

$A_{L}=118.5 \rightarrow 111.5$ to the measured data we extract a dissipated energy of order $10 \mathrm{MeV}$ for ${ }^{237} \mathrm{~Np}$. It is interesting to note that our estimate of $E_{\text {diss }}=10$ $\mathrm{MeV}$ is compatible with results from other low-energy fission measurements that are based on the odd-even effect in the charge yields of fission fragments 12,13 . 

7. V.E. Oberacker, A.S. Umar, J.C. Wells, C. Bottcher and M.R. Strayer, Phys. Lett. B 293, 270 (1992).

8. B.B. Back, Nucl. Phys. A 228, 323 (1974).

9. W.U. Schröder, W.W. Wilcke, M.W. Johnson, D. Hilscher, J. R. Huizenga, J. C. Browne and D. G. Perry, Phys. Rev. Lett. 43, 672 (1979).

10. K.T.R. Davies, A.J. Sierk and J.R. Nix, Phys. Rev. C 13, 2385 (1976).

11. F. Risse et al., Z. Phys. A 339, 427 (1991).

12. C. Wagemans, The Nuclear Fission Process, p.418 (CRC Press, Boca Raton, 1991)

13. F. Goennenwein, private communication at this Conference 
M98004059

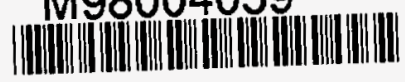

Report Number (14)ORNL/CP - 06416

$20 N F-9711113 \ldots$

Publ. Date (11) $\quad 199801$

Sponsor Code (18) $\frac{\text { DUElER, XP }}{U C-400, D O E / E R}$
UC Category (19)

DOE 